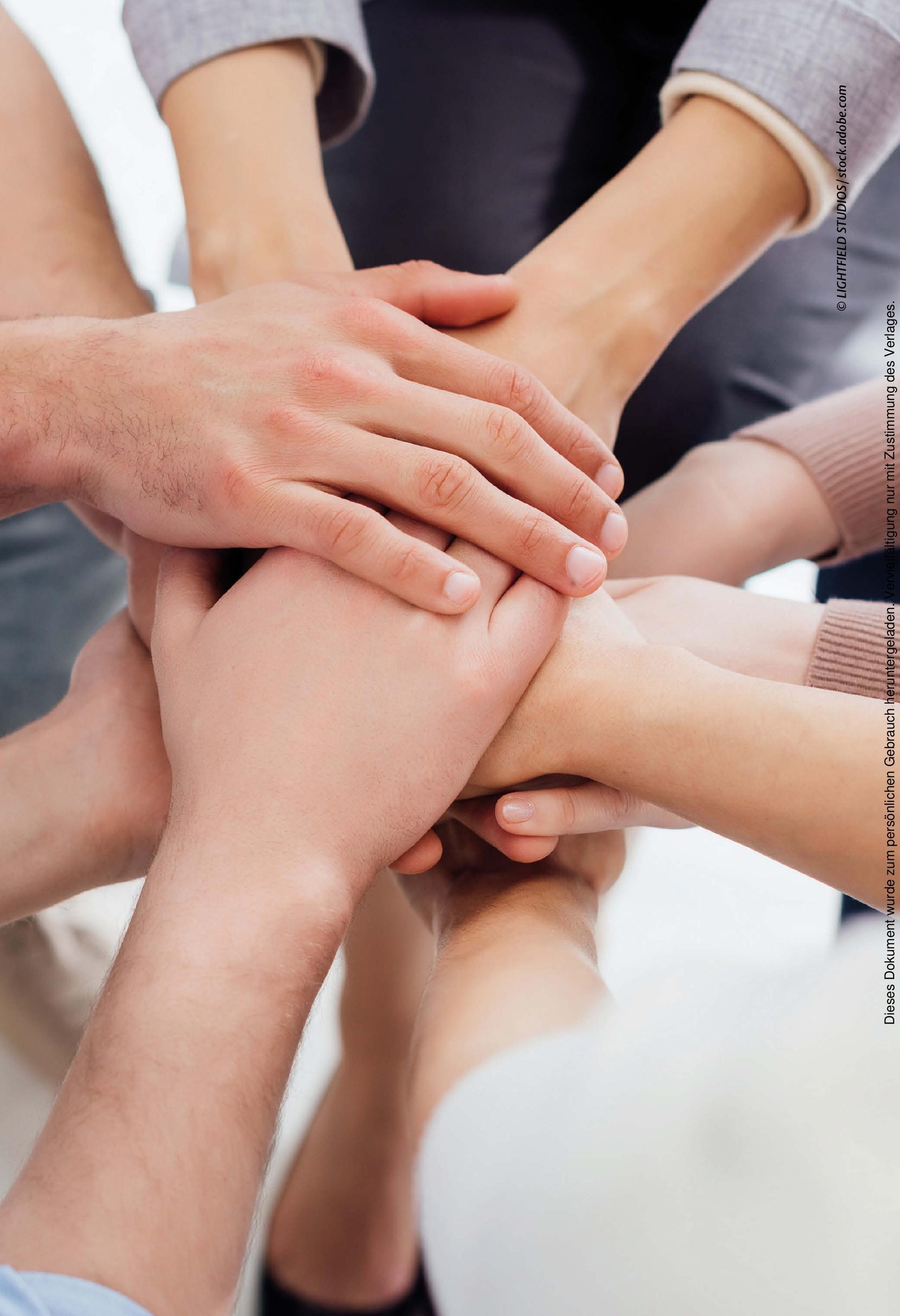




\title{
Hand in Hand für den Berufsstand
}

\begin{abstract}
Seitdem Bundesgesundheitsminister Jens Spahn 2019 ein Rechtsgutachten zum Heilpraktikerrecht in Auftrag gegeben hat, geht eine Welle der Verunsicherung durch die Reihen der Heilpraktiker und Heilpraktikeranwärter. Werden wir jetzt abgeschafft, soll das Heilpraktikergesetz reformiert werden, wird die Ausbildung akademisiert - das sind nur einige der Spekulationen. Fest steht: Die Zeichen stehen auf Veränderung. In welcher Form und in welchem Ausmaß, ist bisher ungewiss. Ein Interview mit HP Elvira Bierbach und HP Christian Blumbach, dem Sprecherteam der Gesamtkonferenz der Heilpraktikerverbände und Fachgesellschaften.
\end{abstract}

Frau Bierbach, Herr Blumbach, warum braucht Deutschland seine Heilpraktiker?

Elvira Bierbach: Heilpraktikerinnen und Heilpraktiker sind in Ergänzung zum ärztlichen Behandlungsspektrum tätig. Sie bieten ihren Patientinnen und Patienten neben all ihren bewährten traditionellen und modernen Therapieverfahren und den ursächlichen, ganzheitlichen und konstitutionellen Behandlungsansätzen auch Zuwendung, Menschlichkeit und Zeit. Je schneller und effizienter der ärztliche Betrieb Behandlungen durchführen muss, desto größer wird der Wunsch der Patienten nach Beratung und Behandlung durch Heilpraktiker. Dieser Prozess vollzieht sich schon seit vielen Jahrzehnten. Der demografische Wandel und der in vielen Regionen Deutschlands jetzt schon bestehende gravierende Ärztemangel werden die medizinische Versorgung der Bevölkerung zudem weiter erschweren. Auch im Hinblick auf diese Entwicklung sollte die Stellung des Heilpraktikerberufs im Gesundheitssystem gestärkt werden!

Die Weltgesundheitsorganisation forderte alle Länder weltweit dazu auf, ihre jeweilige traditionelle und komplementäre Medizin in die nationalen Gesundheitssysteme zu integrieren. Bisher ist Deutschland dem nicht nachgekommen. Wir Heilpraktiker bewahren unsere naturheilkundlichen Traditionen auch als wichtiges Kulturgut. In Deutschland wirkten bedeutende Heilerpersönlichkeiten, die weltweit Ruf erlangt haben und deren Verständnis von Krankheit und Heilung heute unersetzlicher Teil des immateriellen Erbes ist. Nehmen Sie zum Beispiel Hildegard von Bingen, Samuel Hahnemann oder Sebastian Kneipp. Darüber hinaus integrieren unsere Verfahren das Erbe anderer Kulturen, hier sind etwa der Ayurveda oder die TCM zu nennen. Und letztendlich dürfen wir nicht vergessen: Die Wahl des Therapeuten fällt unter das Grundrecht auf freie Weltanschauung.
Christian Blumbach: Den Bedarf an komplementärer Medizin sehen wir auch in allen anderen europäischen Ländern. Überall dort arbeiten Kollegen von uns, zumeist aber auf rechtlich tönernen Füßen. In Deutschland herrschte bislang eine relativ sichere Rechtslage. Doch durch das vor einigen Jahren in Kraft getretene Gesetz zur Patientensicherheit sind jetzt Modernisierungen nötig geworden. Sie sind notwendig, um die Naturheilkunde für künftige Generationen von Heilpraktikern und Patienten zu sichern.

Wie wahrscheinlich ist es, dass die Bundesregierung eine Reform des Heilpraktikerberufs plant? Und wie realistisch wäre ein Verbot des Berufs?

Elvira Bierbach: Alle Gesundheitsberufe Deutschlands werden reformiert. Das steht schon im aktuellen Koalitionsvertrag und wird auch nach der Bundestagswahl erklärtes Ziel bleiben. Das 2019 von Jens Spahn in Auftrag gegebene Rechtsgutachten zum Heilpraktikerrecht ist noch nicht öffentlich, und das kann gemäß aktueller Auskunft vom Bundesgesundheitsministerium noch andauern ... Wir sind alle sehr gespannt auf den Inhalt.

Der BDH e.V. hat seinerseits mit Unterstützung des VfP e.V. und des VUH e.V. ein Rechtsgutachten zu den gleichen Fragestellungen bei einem sehr renommierten Verfassungsrechtler, Prof. Dr. Helge Sodan, in Auftrag gegeben (Anmerkung der Redaktion: siehe S. 14, 72). Dieses kommt zu dem eindeutigen Schluss, dass ein Totalverbot des Berufs aus verfassungsrechtlicher Sicht kaum durchführbar wäre. Dieses Gutachten fordert eher das Gegenteil, nämlich einen größeren rechtlichen Schutz der Heilpraktikerinnen und Heilpraktiker. Eine Möglichkeit für mehr rechtliche Sicherheit wäre die Bildung einer Kammer.

Christian Blumbach: Eine solche Regelung hat Vor- und Nachteile, bedeutet mehr Rechte, aber auch mehr Pflichten. Bei Kammerberufen gibt es beispielsweise eine Zwangsmitgliedschaft für alle 
Berufsangehörigen, eine berufsständische Ausbildungsregelung und Qualitäts- und Selbstkontrolle, aber auch größere Rechtssicherheit. Die Idee einer Verkammerung ist rechtswissenschaftlich bestimmt richtig, aber nicht unumstritten. Es scheint in der Kollegenschaft auf wenig Gegenliebe zu stoßen. Dieses Rechtsgutachten wurde in Berlin der Politik vorgestellt, und wir als Vertretung der Gesamtkonferenz waren mit eingeladen.

Wie schnell ist mit weiteren Maßnahmen der Bundesregierung bezüglich des Heilpraktikerberufs zu rechnen?

Christian Blumbach: Eine Einschätzung ist hier schwer zu treffen ... Wir vermuten und hoffen, dass es von politischer Seite vor der Bundestagswahl keine gravierenden Aktionen bezüglich unseres Berufsstandes mehr geben wird - doch nach der Regierungsbildung könnte es dann sehr rasch vorangehen.

Wir befinden uns in einem Super-Wahljahr. Natürlich kann es aufgrund der Corona-Pandemie noch zu Terminverschiebungen kommen, aber aktuell sind neben der Bundestagswahl am 26. September 2021 noch fünf Landtagswahlen sowie die Wahl des Berliner Abgeordnetenhauses und zwei Kommunalwahlen geplant. Das Wahljahr kann eine riesige Chance sein, wenn es uns gelingt, eine effektive Öffentlichkeitsarbeit zu machen - und wenn wir es schaffen, den Parteien aufzuzeigen, dass ein ganz erheblicher Teil von Wählerinnen und Wählern unsere Arbeit befürwortet und weiterhin uneingeschränkt nutzen will.

Wie ist denn die Position einzelner Parteien zum Heilpraktikerberuf, können Sie uns Beispiele nennen?

Elvira Bierbach: Ein wichtiges Beispiel für die Heilpraktiker ist die Partei Bündnis 90/Die Grünen: Viele aus unserem Berufsstand wählen traditionell die Grünen. Leider jedoch ist gerade diese Partei mittlerweile diejenige, von der aktuell der größte Gegenwind kommt. Vielfach wird so argumentiert, dass wir nur mit „unwissenschaftlichen Methoden“ arbeiten würden und keine Unterstützung erwarten könnten von einer Partei, die sich bei der Bekämpfung des Klimawandels auf wissenschaftliche Erkenntnisse stützt. Unserer Meinung nach müssen wir insbesondere die junge beziehungsweise an Natur und Artenvielfalt interessierte Wählerschaft über diese Situation aufklären, denn auf diese fokussieren sich die Wahlkampagnen der Grünen. Wir müssen diese Wähler dazu bewegen, sich für unseren Berufsstand auszusprechen. Da liegt noch ein hartes Stück Arbeit vor uns.

Innerhalb der CDU/CSU-Fraktion sind uns viele Abgeordnete wohlgesonnen, und einige unterstützen unsere Anliegen explizit. Dennoch gibt es auch aus diesen Reihen nicht unerheblichen Gegenwind. Die FDP-Fraktion hat sich nach internen Diskussionen mehrheitlich auf unsere Seite geschlagen. Auch von der Partei DIE LINKE kommt aktive Unterstützung. Die SPD zeigt sich momentan noch unentschlossen. Extrem ungünstig ist es, dass die AfD aktuell versucht, sich über unseren Berufsstand zu positionieren dass diese Partei mit einem Antrag zur Heilpraktikerausbildung vorgeprescht ist, könnte uns noch sehr schaden.
In den nächsten Monaten werden wir uns massiv dafür einsetzen, dass wir gerade von den Politikerinnen und Politikern, die zur Wahl stehen, wahrgenommen werden und das tun, was existenziell ist für unseren Berufsstand: Lobbyarbeit!

Wie kann so eine Lobbyarbeit aussehen?

Christian Blumbach: Eigentlich haben wir bereits beste Voraussetzungen dafür geschaffen. Es gibt von verschiedenen politischen Fraktionen die klare Aufforderung, sich proaktiv am Gestaltungsprozess zu beteiligen. Erfreulich viele Politikerinnen und Politiker zeigen sich offen für unsere Anliegen, hier insbesondere die Wahrung unserer Therapiefreiheit und -vielfalt. Abgeordnete und andere Funktionsträger sind sowohl für die Naturheilkunde aufgeschlossen als auch für die Heilpraktikerschaft und wollen uns aktiv unterstützen. Sie fragen uns konkret: Was wollt Ihr als Berufsstand? Wofür genau sollen wir uns stark machen? Aber sie melden uns auch zurück, dass die Vielzahl der Verbände sie irritiert, zumal wenn diese verschiedene Forderungen stellen. Sie erwarten von uns das, was sie von anderen Interessengruppen kennen: Die unterschiedlichen Meinungen werden im Vorfeld geklärt, das Ergebnis gebündelt und dann als gesammelte Forderung von gewählten Vertretern vorgetragen.

Elvira Bierbach: Zu unserer Lobbyarbeit-Strategie gehört, für alle eventuell anstehenden Diskussionspunkte konkrete Konzepte auszuarbeiten, die wir bedarfsgerecht in die Entscheidungsprozesse der Politik einfließen lassen können. Das Wichtigste wäre deshalb, dass die Verbände sich um Konsens bemühen und zum Wohle des Berufsstands nach außen geschlossen auftreten. Es ist schön, dass sich so viele Kolleginnen und Kollegen in verschiedenen Konstellationen zusammenfinden, um über unterschiedlichste Facetten des Berufs zu diskutieren. Von der Vielfalt profitiert unser Beruf. In allen menschlichen Beziehungen gibt es verschiedene Ansichten - das liegt in der Natur der Sache. Man sollte diese aber intern besprechen, demokratisch abstimmen und bei den Kernthemen nach außen hin geschlossen eine Meinung vertreten.

Abgesehen von Lobbyarbeit - welche konkreten Maßnahmen sind nötig, um die Zukunft des Berufsstands zu sichern?

Elvira Bierbach: In den letzten Jahren waren wir viel zu oft in der Verteidigungsposition - wir haben reagiert auf diffamierende Fernsehberichte, verunglimpfende Zeitungsartikel und Verleumdungen bestimmter Skeptiker. Wir haben uns auch juristisch wehren müssen, wenn unsere Berufsfreiheit eingeschränkt wurde, zum Beispiel beim Verbot der Eigenbluttherapie oder aktuell, um zu verhindern, dass bestimmte Formulierungen im Entwurf des MTA-Reformgesetzes zukünftig unsere Möglichkeiten der Labordiagnostik massiv einschränken.

Wir sollten mit aller Kraftanstrengung raus aus der Verteidigungsposition. Neben all der notwendigen Lobbyarbeit sollten wir zur genauen Betrachtung unseres Berufsstands bereit sein und schauen, wo Modernisierung im Sinne von Patientenschutz und Gemeinwohl zeitgemäß und angemessen ist. Entsprechend sollten wir proaktiv und freiwillig leicht in der Praxis umsetzbare 
Konzepte vorschlagen. Denn Patientenschutz und Gemeinwohl sind den Politikern, genau wie uns, äußerst wichtig.

Christian Blumbach: Und Konzepte gibt es längst! Ein Beispiel: Die Politik fordert von uns Qualitätssicherung, dafür haben wir bereits etablierte Werkzeuge - von Schulungen und hochkarätigen Fachausbildungen bis hin zu einem Fortbildungszertifikat für Heilpraktiker, welches Zigtausende von Kolleginnen und Kollegen nutzen. Wir kommunizieren dies jedoch viel zu wenig - weder gegenüber den Medien, noch gegenüber der Politik. Das macht uns angreifbar, und deshalb muss das anders werden.

Was hilft dem Berufsstand aus Ihrer Sicht nicht?

Elvira Bierbach: Sicherlich ist es nicht hilfreich, in einem Atemzug mit Reichsbürgern und QAnon-Anhängern genannt zu werden. Die ausufernden Proteste vor dem Reichstag haben uns massiv in ein schlechtes Licht gerückt, und das nur, weil eine Heilpraktikerin in vorderster Front mit dabei war. Ab da haben viele Medien den Heilpraktiker unter den Generalverdacht der Coronaleugnung bis hin zur rechten Gesinnung und Antisemitismus gerückt (Anmerkung der Redaktion: siehe S. 79). Selbstverständlich haben wir uns in all diesen Fällen gewehrt, uns ausdrücklich von extremistischen, undemokratischen und menschenverachtenden Bewegungen distanziert und den Sachverhalt richtiggestellt - die negativen Schlagzeilen waren aber natürlich in der Welt.

Wir Heilpraktiker sind weltoffen, schließlich praktizieren wir Methoden aus allen möglichen Kulturen und Weltanschauungen. Humanität, Wertschätzung, Respekt und Menschenliebe bilden eine Grundlage unserer Arbeit. Unsere ganze Kollegenschaft ist salopp gesagt - eine bunte Mischung unterschiedlichster Menschen. Deshalb setzen wir ein klares Zeichen: Wir Heilpraktiker sind bunt, nicht braun.

\section{Was kann jeder Einzelne tun?}

Christian Blumbach: Jeder Heilpraktiker kann seine Patienten dazu mobilisieren, sich zu unserem Berufsstand zu bekennen. Unsere Patientinnen und Patienten schätzen unsere Arbeit und vertrauen uns. Wir stellen oft fest, dass sie zum Beispiel durch negative Berichterstattung nicht verunsichert sind, sondern zu uns stehen. Hinter uns stehen also Millionen von Menschen, die wir aktivieren könnten. Wir können sie für Abstimmungen oder Aktionen gewinnen und so massiv die Bemühungen der Heilpraktikerverbände mit Masse unterstützen. Bei über 40 Millionen Patientenkontakten pro Jahr käme einiges an Resonanz zusammen. Ganz konkret: Die Change.org-Petition „Petition für den Erhalt des Heilpraktikerberufes in seiner gesamten Vielfalt“ hat mittlerweile etwa 202000 Unterschriften gezeichnet. Das ist kein schlechtes Ergebnis, aber mit mehr Einsatz könnten es noch bedeutend mehr werden - und das wäre ein eindrucksvolles Signal an die Politik.

Die Gesamtkonferenz sucht auch Kolleginnen und Kollegen, die sich ehrenamtlich in unserem Team engagieren und uns bei gezielten Aktionen unterstützen. Der Zeitaufwand ist überschaubar und wird selbst bestimmt. Für die Kolleginnen und Kollegen ist es ein gutes Gefühl, aktiv bei der Sicherung des Berufsstands mitwirken zu können. Interessierte bitten wir herzlich um Kontaktaufnahme für weitere Informationen.

Eine einfache Möglichkeit, sich für unseren Berufsstand einzusetzen, sind Spenden: PR-Kampagnen, Öffentlichkeitsarbeit - all das kostet viel Geld, und das Budget vieler Verbände ist leider recht klein. Deshalb bitten wir die Kollegenschaft und auch unsere Patienten um finanzielle Unterstützung - jeder Betrag hilft (Anmerkung der Redaktion: Weitere Informationen unter: www.gesamtkonferenz-heilpraktiker.de/unterstuetzen/).

Sie sprachen gerade von der Gesamtkonferenz. Bitte erklären Sie unseren Lesern, worum es bei der Gesamtkonferenz geht.

Elvira Bierbach: So vielfältig wie unser Berufstand ist, so vielfältig sind auch die verschiedenen Interessenvertretungen der Heilpraktikerschaft. Manche Verbände haben vier oder fünf Dutzend Mitglieder, andere Tausende. Jeder Verband engagiert sich auf seine Weise bestmöglich für seine Mitglieder - und das hat über Jahrzehnte auch gut funktioniert. Doch seit einigen Jahren gibt es gezielte Bestrebungen extrem gut organisierter und vernetzter lobbyistischer Interessensvertretungen, unseren Berufstand massiv zu beschneiden oder gar ganz abzuschaffen. Da können selbst die besten Aktionen einzelner Verbände kaum etwas entgegensetzen. Hier hilft nur eins: Kräftebündelung!

Christian Blumbach: Deshalb arbeiten wir seit zwei Jahren mit der Gesamtkonferenz daran, möglichst viele Verbände und Fachgesellschaften an einen Tisch zu bekommen und alle Aktionen, die im Zusammenhang mit politischer Wahrnehmung der Heilpraktikerschaft zu tun haben, zu koordinieren beziehungsweise in Einklang zu bringen. Auf diesem Weg haben wir, trotz anfänglicher Skepsis, sehr beeindruckende Erfolge zu verzeichnen: Mittlerweile bekennen sich 32 Interessenvertretungen der Heilpraktikerschaft zur Idee der Gesamtkonferenz, und dahinter steht eine entsprechend große Anzahl von Kolleginnen und Kollegen. Die Verbändevertretungen sind im Austausch und treffen sich in regelmäßigen Konferenzen. Leider konnten wir noch nicht alle Heilpraktikerverbände überzeugen, doch lassen wir nicht locker und laden immer wieder ausdrücklich alle Verbände und Fachgesellschaften herzlich ein. Jeder Verband bewahrt seine Eigenständigkeit und seinen Charakter. Und natürlich wird diskutiert, auch gestritten und um Ergebnisse gerungen. Gerade für die Erhaltung unserer Freiheit und Vielfalt ist es unverzichtbar, dass wir uns solidarisch und transparent austauschen, uns abstimmen und gegenseitig unterstützen, um am Ende mit einer Stimme sprechen.

Wie kann der Heilpraktikerberuf der Zukunft aussehen: Was wäre ein bestmöglicher Ausgang der momentanen Entwicklungen, was das denkbar schlechteste Ergebnis?

Christian Blumbach: Optimal für uns wäre, dass sich am momentanen Status nichts ändern würde. Aber das ist realistisch betrachtet nicht zu erwarten! Umso wichtiger ist es, dass wir alles dafür tun, die größtmögliche Freiheit in unserem so breit gefächerten und vielseitigen Beruf zu erhalten.

Die denkbar schlechteste Entwicklung wäre ein Verbot oder die Einschränkung einzelner Behandlungsverfahren und/oder 
eine Akademisierung der Ausbildung und damit ein Aushöhlen unseres Berufs. Eine gute Ausbildung ist wichtig, aber eine Reform sollte - und da sind sich alle Verbände einig - nicht in Richtung einer Akademisierung gehen. Jeder sollte die Möglichkeit haben, seine naturheilkundlichen Schwerpunkte selbst zu wählen und sich nicht in einem Studium wiederfinden, das zu einem Berufsprofil eines „kleinen Hausarztes“ führen würde. Ein anderes Horrorszenario wäre eine Art „Genesungspraktiker“, der nur auf ärztliche Weisung hin tätig werden darf.

Das aktuelle System verbietet kein Denken abseits ausgetretener Pfade und ist nicht dogmatisch. Jeder Kollege hat das Recht und die Freiheit, seinen eigenen Weg zu gehen. Dabei verwenden wir wirkungs- und kraftvolle Methoden verschiedenster Heilsysteme. Dieses System muss nach Möglichkeit erhalten bleiben.

Dieser Artikel ist online zu finden:

http://dx.doi.org/10.1055/a-1325-8964

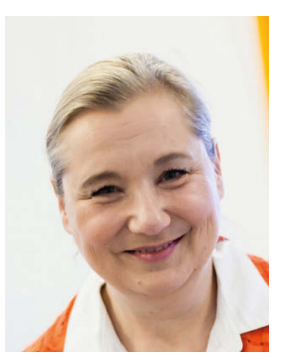

\section{HP ELVIRA BIERBACH}

Elvira Bierbach ist Heilpraktikerin und leitet seit 1992 eine renommierte Heilpraktikerschule in Bielefeld. Außerdem hat sie verschiedene Lehrbücher für Heilpraktiker(-anwärter) herausgegeben, darunter das bekannte Buch „Naturheilpraxis heute“, das Standardlehrwerk für alle HPAs, und hält Vorträge rund um die Heilpraktikerausbildung. Seit 2001 ist sie Beiratsmitglied im „Bund Deutscher Heilpraktiker (BDH)“ und seit 2006 die hauptverantwortliche Herausgeberin der Deutschen Heilpraktiker Zeitschrift DHZ. Darüber hinaus ist sie Sprecherin der „Gesamtkonferenz Deutscher Heilpraktikerverbände und Fachgesellschaften" sowie 1. Vorstand des „HeilpraktikerNetzwerks", das sich aus der Gesamtkonferenz entwickelt hat.

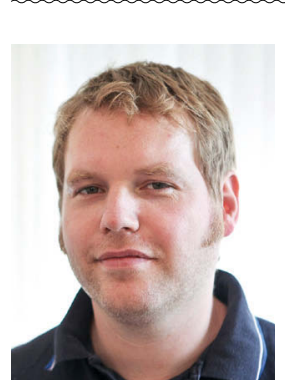

\section{HP CHRISTIAN BLUMBACH}

Christian Blumbach ist seit 17 Jahren in eigener Praxis in Duisburg tätig. Seit 11 Jahren ist er zudem 1. Vorsitzender der ACON e. V. (Arbeitsgemeinschaft für Chiropraktik, Osteopathie und Neuraltherapie Deutscher Heilpraktiker e. V.). Darüber hinaus ist er Sprecher „Gesamtkonferenz Deutscher Heilpraktikerverbände und Fachgesellschaften" sowie 2. Vorstand des "Heilpraktiker-Netzwerks", das sich aus der Gesamtkonferenz entwickelt hat.

\section{ERFAHREN SIE MEHR ZUR AKTUELLEN BERUFS- POLITISCHEN SITUATION BEIM 37. DEUTSCHEN HEILPRAKTIKERTAG!}

Wie ist aktuell die politische Entwicklung? Wie ist die Stimmung in Berlin, in den Landeshauptstädten? Will man den Heilpraktikerberuf dort noch - oder eher nicht? Wie könnte das Rechtsgutachten der Bundesregierung ausfallen - best case und worst case? Was kann und muss der Berufsstand tun, um seine Zukunft zu sichern?

Darüber sprechen Ulrich Sümper und Siegfried Kämper, Präsident und Vizepräsident des Bundes Deutscher Heilpraktiker $(B D H)$, in ihrem Vortrag „Alles aus? Ein Update zur politischen Situation des Heilpraktikerberufs“.

Der Vortrag findet am 15. Mai 2021 im Congress Center Düsseldorf statt und wird auch live gestreamt. Weitere Infos unter www.thieme.de/de/heilpraxis/deutscher_heilpraktikertag66829.htm und bei der Anmeldung zum DHT. 